\title{
2D transmission profile of light through highly scattering media
}

\author{
T. Sperling, W. Bührer, C. M. Aegerter, G. Maret \\ Department of Physics, University of Konstanz, Germany
}




\section{D transmission profile of light through highly scattering media}

T. Sperling, W. Bührer, C. M. Aegerter, G. Maret Department of Physics, University of Konstanz, Germany

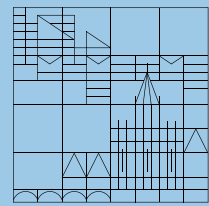

\section{$=-x+1$}

\section{Theory}

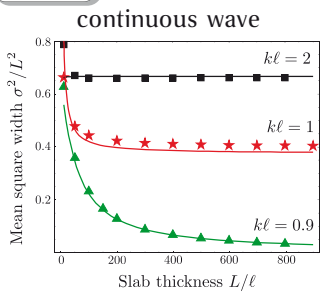

Slab thickness $L / \ell$

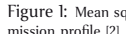

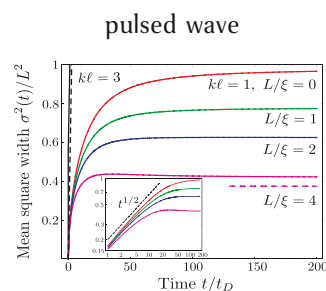

Figure 2: Mean square
Analytically, it was shown that the mean square width $\sigma^{2}$ of the transmission profile of a focused monochromatic beam through disordered media is [2]:

\begin{tabular}{c||c|c} 
& $k l^{*}$ & $\sigma^{2}$ \\
\hline \hline diffusion & $k l^{*} \gg 1$ & $\frac{2}{3} L^{2}-\mathcal{O}\left(\left(l^{*}\right)^{2}\right)$ \\
mobility edge & $k l^{*}=1$ & $\frac{3}{8} L^{2}+\mathcal{O}\left(L z_{c}\right)$ \\
localisation & $k l^{*}<1$ & $2 L \xi+\mathcal{O}\left(\xi^{2}\right)$
\end{tabular}

\section{Experimental setup

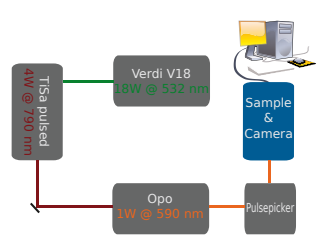 \\ Setup -250 fs pulses @ $590 \mathrm{~nm}$ - Pulsepicker contrast up to $500: 1$ \\ - 16 bit camera, $512 \times 512$ pixel - Minimum gatingtime $1 \mathrm{~ns}$ - 250 ps time resolution \\ Samples \\ \begin{tabular}{c|c|c||c|} 
Sample & $d(\mathrm{~nm})$ & Disp. (\%) & $k l^{*}$ \\
\hline \hline R104 & 311 & &
\end{tabular}

\begin{tabular}{c|c|c||c|} 
R104 & 311 & 10 & $3.5-4$ \\
R700 & 230 & 22 & 2.5
\end{tabular} \begin{tabular}{l|l|l|l} 
R902 & 279 & 38 & 4.3
\end{tabular}}

\section{Data evaluation}

The measured 2D transmission profile is fitted with a $2 \mathrm{D}$ gaussian. The mean square width is averaged to $\sigma^{2}$ and evaluated.

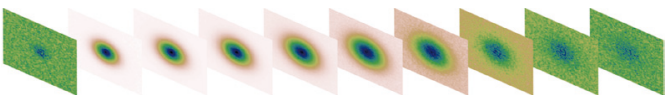

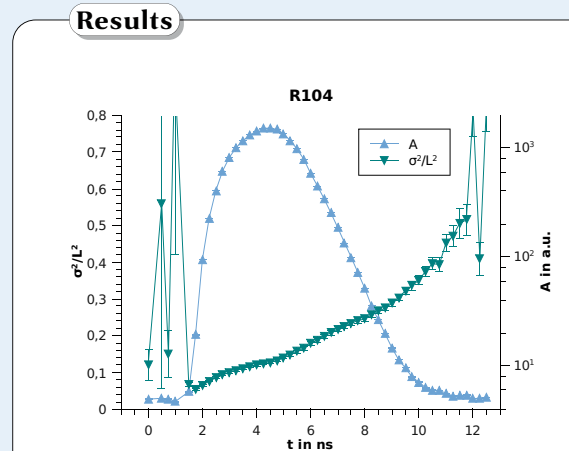
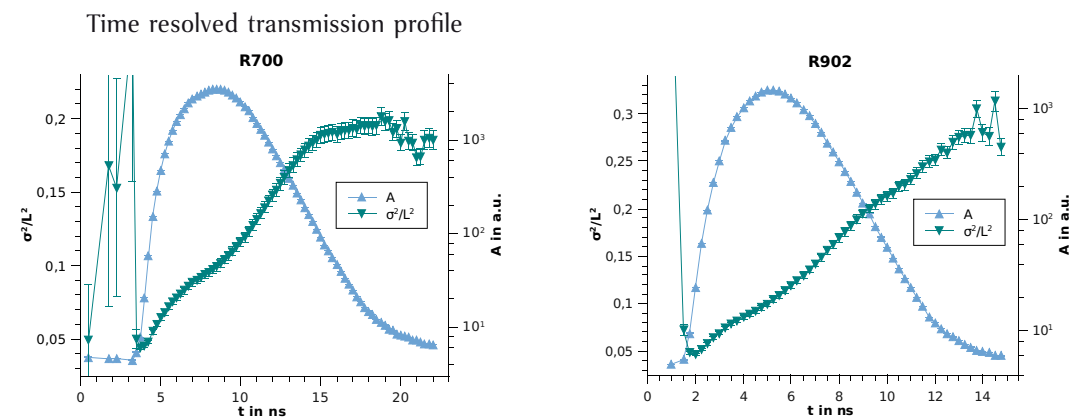

Figure 4: $\frac{\sigma^{2}}{T^{2}}$ measured time dependent for three different samples. Obviously there is plateau for the R700 sample. For the R104 sample no plateau was measured. The pulse disappeared in noise before the theoretically value for diffusive media of $\frac{\sigma^{2}}{L^{2}}=\frac{2}{3}$ could be reached. R902 indicates a plateau at $\frac{\sigma^{2}}{L^{2}}=0.28$, but the intensity is very low and the afterpulse appears around $15.5 \mathrm{~ns}$. From theory it is expected that the plateau shows up at $\frac{\sigma^{2}}{L^{2}}=2 \frac{\xi}{L}$. For R700 the $L=1.01 \mathrm{~mm}$ thick sample the diffusion constant is $D=13 \frac{\mathrm{m}^{2}}{\mathrm{~s}}$, the absorption time $\tau_{\text {abs }}=1.7 \mathrm{~ns}$ and the localisation time $\tau_{\mathrm{loc}}=4.9 \mathrm{~ns}$. The plateau should be at $\frac{\sigma^{2}}{L^{2}}=0.5$, but is located at $\frac{\sigma^{2}}{L^{2}}=0.193$.

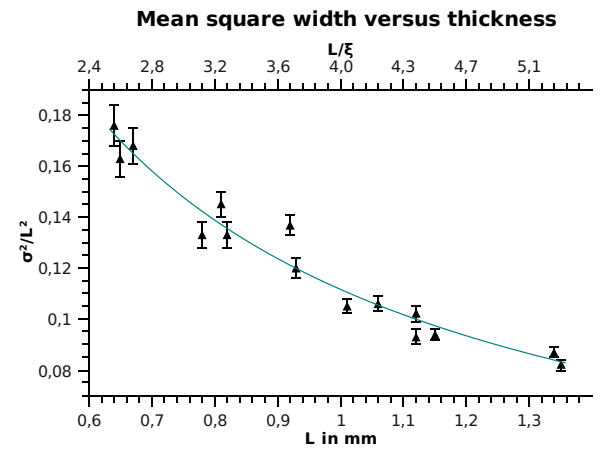

Figure 5: Measurement of $\frac{\sigma^{2}}{T^{2}}$ with R700 in static transmission with $\xi=0.252 \mathrm{~mm}$. The fit results of $\frac{\sigma^{2}}{L^{2}}=A \cdot L^{a}$ are: $A=0.117 \mathrm{~mm} \pm 0.002 \mathrm{~mm}$ and $a=-0.976 \pm 0.060$.

The fit parameter A is equal to $\lambda$. With $\frac{1}{\lambda}=\frac{1}{\xi}+\frac{1}{l_{a}}, \xi=0.252 \mathrm{~mm}$ and $l_{a}=0.149 \mathrm{~mm}$, it is $\lambda=0.094 \mathrm{~mm}$, which is very close to A. The exponent $a$ should scale with $a=-1$, because it should be $\frac{\sigma^{2}}{L^{2}} \propto \frac{1}{L l_{e}}$, which is clearly shown.

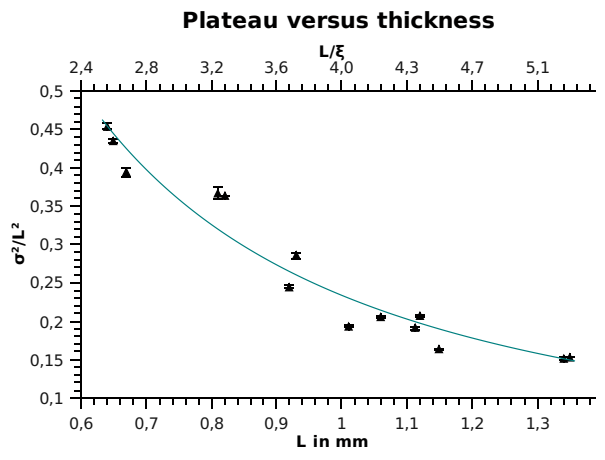

Figure 6: Time resolved measurement of the plateau (see fig. 4) of $\frac{\sigma^{2}}{L^{2}}$ in transmission with R700 and $\xi=0.252 \mathrm{~mm}$. The results of a fit with $\frac{\sigma^{2}}{L^{2}}=A \cdot L^{a}$ are: $A=0.237 \pm 0.008$ and $a=-1.491 \pm 0.112$.

The fitted exponent of $a=-1.491 \pm 0.112$ does not match the expected value of $a=-1$. It is not obvious why the exponent should be approximately $-\frac{3}{2}$.

\section{References}

II] P.W. Anderson: The question of classical localization: a theory of white paint?, Phil. Mag. B 52, 505 (1985)

[2] N. Cherroret, SE Skipetrov, B van Tiggelen: Transverse confinement of waves in 3D random media, arXiv:0810.0767v2 (2008), accepted in Phys. Rev . E

Thight Doctoral dissertation, University of Konstanz (2006)

[4] C. M. Aegerter, M. Störzer and G. Maret: Experimental determination of critical exponents in Anderson localisation of light, Europhys. Lett., 75, 562 (2006) 